\title{
A handy approximation for a mediated bioelectrocatalysis process, related to Michaelis-Menten equation
}

\author{
Uriel Filobello-Nino' ${ }^{1}$, Hector Vazquez-Leal ${ }^{1 *}$, Brahim Benhammouda ${ }^{3}$, Luis Hernandez-Martinez², \\ Yasir Khan ${ }^{4}$, Victor Manuel Jimenez-Fernandez ${ }^{1}$, Agustin Leobardo Herrera-May ${ }^{5}$, Roberto \\ Castaneda-Sheissa ${ }^{1}$, Domitilo Pereyra-Diaz' ${ }^{1}$ Juan Cervantes-Perez ${ }^{1}$, Jose Antonio Agustin \\ Perez-Sesma ${ }^{1}$, Sergio Francisco Hernandez-Machuca ${ }^{1}$ and Leticia Cuellar-Hernandez ${ }^{1}$
}

\begin{abstract}
In this article, Perturbation Method (PM) is employed to obtain a handy approximate solution to the steady state nonlinear reaction diffusion equation containing a nonlinear term related to Michaelis-Menten of the enzymatic reaction. Comparing graphics between the approximate and exact solutions, it will be shown that the PM method is quite efficient.
\end{abstract}

Keywords: Michaelis-Menten kinetics; Perturbation method; Reaction/diffusion equation; Mediated bioelectrocatalysis

\section{Introduction}

Michaelis-Menten equation is used to describe the kinetics of enzyme-catalyzed reactions for the case in which the concentration of substrate is grater than the concentration of enzyme. These reactions are important in biochemistry because the most of cell processes require enzymes to obtain a significant rate (Michaelis and Menten 1913; Murray 2002). Enzymes are large protein molecules, which act as remarkably catalyst to speed up chemical reactions in living beings. With this end, they do work on specific molecules, called substrates; without the presence of enzymes, the majority of chemical reactions that keep living things alive would be too slow to maintain life (Michaelis and Menten 1913).

As it was already mentioned, the aim of this study is to find a handing approximate solution which best describes a reaction diffusion process related to Michaelis-Menten kinetics. Several oxidoreductase reactions such as quinones and ferrocenes consist of electrode reactions which allow conjugating between redox enzyme

\footnotetext{
*Correspondence: hvazquez@uv.mx

1 Electronic Instrumentation Faculty, Universidad Veracruzana, Cto. Gonzalo

Aguirre Beltran S/N, 91000 Xalapa, Mexico

Full list of author information is available at the end of the article
}

reactions and electrode reactions. The redox compoundmediated and enzyme catalysed electrode process is called mediated bioelectrocatalysis (Thiagarajan et al. 2011). Among its applications in engineering it is utilized for biosensors, bioreactors, and biofuel cells. Therefore, it is important the search for accurate solutions for this equation. Unfortunately, solving nonlinear differential equations is not a trivial process.

The Perturbation Method (PM) is a well established method; it is among the pioneer techniques to approach various types of nonlinear problems. This procedure was originated by S. D. Poisson and extended by J. H. Poincare. Although the method appeared in the early 19th century, the application of a perturbation procedure to solve nonlinear differential equations was performed later on that century. The most significant efforts were focused on celestial mechanics, fluid mechanics, and aerodynamics (Chow 1995; Filobello-Nino et al. 2013; Holmes 1995).

In general, it is assumed that the differential equation to be solved can be expressed as the sum of two parts, one linear and the other nonlinear. The nonlinear part is considered as a small perturbation represented by a small parameter (the perturbation parameter). The assumption that the nonlinear part is small compared to the linear

\section{Springer}

(c) 2014 Filobello-Nino et al: licensee Springer. This is an Open Access article distributed under the terms of the Creative

Commons Attribution License (http://creativecommons.org/licenses/by/2.0), which permits unrestricted use, distribution, and reproduction in any medium, provided the original work is properly credited. 
is considered as a disadvantage of the method. There are other modern alternatives to find approximate solutions to differential equations describing some nonlinear problems such as those based on: variational approaches (Assas 2007; He 2007; Kazemnia et al. 2008; Noorzad et al. 2008), Tanh method (Evans and Raslan 2005), expfunction (Mahmoudi et al. 2008; Xu 2007), Adomian's decomposition method (Adomain 1988; Babolian and Biazar 2002; Chowdhury 2011; Jiao et al. 2001; Kooch and Abadyan 2011,2012; Vanani et al. 2011), parameter expansion (Zhang and $\mathrm{Xu}$ 2007), homotopy perturbation method (Beléndez et al. 2009; Biazar and Aminikhan 2009; Biazar and Ghazvini 2009; El-Shaed 2005; Fathizadeh et al. 2011; Faraz and Khan 2011; Feng et al. 2007; Fereidoon et al. 2010; Filobello-Nino et al. 2012a, 2012b; Ganji et al. 2008, 2009; He 1999, 2000a, 2006a, 2006b, 2008; Hossein 2011; Khan et al. 2011, 2013; Madani et al. 2011; Mirmoradia et al. 2009; Noor and MohyudDin 2009; Sharma and Methi 2011; Thiagarajan et al. 2011; Vazquez-Leal et al. 2012a, 2012b), and homotopy analysis method (Hassana and El-Tawil 2011; Patel et al. 2012), among many others.

Although the PM method provides, in general, better results for small perturbation parameters $\varepsilon<<1$; we will see that our approximation, besides of being handy, has good accuracy even for relatively large values of the perturbation parameter.

The paper is organized as follows. First, we introduce the basic idea of the PM method. Second, we provide an application of the PM method solving the bioelectrocatalysis process already mentioned. Next, we discuss significant results obtained by applying the method. Finally, a brief conclusion is given.

\section{Basic idea of perturbation method}

Let the differential equation of one dimensional nonlinear system be in the form

$$
L(x)+\varepsilon N(x)=0,
$$

where we assume that $x$ is a function of one variable $x=$ $x(t), L(x)$ is a linear operator which, in general, contains derivatives in terms of $t, N(x)$ is a nonlinear operator, and $\varepsilon$ is a small parameter.

Considering the nonlinear term in (1) to be a small perturbation and assuming that its solution can be written as a power series for the small parameter $\varepsilon$

$$
x(t)=x_{0}(t)+\varepsilon x_{1}(t)+\varepsilon^{2} x_{2}(t)+\ldots .
$$

Substituting (2) into (1) and equating terms having identical powers of $\varepsilon$, we obtain a number of differential equations that can be integrated, recursively, to determine the unknown functions: $x_{0}(t), x_{1}(t), x_{2}(t) \ldots$

\section{Approximate solution for the nonlinear reaction/diffusion equation under study}

The equation to solve is

$$
y^{\prime \prime}-\frac{k y}{1+\alpha y}=0,0 \leq x \leq 1, y(0)=1, y(1)=0,
$$

where $k$ and $\alpha$ denote positive reaction diffusion and saturation parameters, respectively, for the mentioned process; $y$ is the mediator concentration and $x$ the distance (Thiagarajan et al. 2011).

It is possible to find a handy solution for (3) by applying the PM method, and identifying terms

$$
\begin{array}{r}
L(y)=y^{\prime \prime}(x), \\
N(y)=-k y(1+\alpha y)^{-1} .
\end{array}
$$

We use Newton's binomial to transform (3) into the following approximate form

$$
\begin{aligned}
y^{\prime \prime}-k y\left(1-\alpha y+\alpha^{2} y^{2}\right) & =0,0 \leq x \leq 1, y(0) \\
& =1, y(1)=0,
\end{aligned}
$$

identifying $\alpha$ as the PM parameter (see (2)), we assume a solution for (6) in the form

$$
y(x)=y_{0}(x)+\alpha y_{1}(x)+\alpha^{2} y_{2}(x)+\alpha^{3} y_{3}(x)+\alpha^{4} y_{4}(x)+\ldots
$$

Equating terms with identical powers of $\alpha$, it can be solved for $y_{0}(x), y_{1}(x), y_{2}(x), \ldots$, and so on. Later on will be seen that a very good handy result is obtained by keeping just the first order approximation.

$$
\begin{array}{ll}
\left.\alpha^{0}\right) & y_{0}^{\prime \prime}-k y_{0}=0, y_{0}(0)=1, y_{0}(1)=0, \\
\left.\alpha^{1}\right) & y_{1}^{\prime \prime}-k y_{1}+k y_{0}^{2}=0, y_{1}(0)=0, y_{1}(1)=0,
\end{array}
$$

The solution for (8) that satisfies the boundary conditions is given by

$$
y_{0}(x)=A e^{\sqrt{k} x}+B e^{-\sqrt{k} x},
$$

where $A$ and $B$ are constants given by

$$
\begin{aligned}
& A=\frac{-1}{e^{2 \sqrt{k}}-1}, \\
& B=\frac{e^{2 \sqrt{k}}}{e^{2 \sqrt{k}}-1} .
\end{aligned}
$$

Substituting (10) into (9), we obtain

$$
\begin{aligned}
y_{1}^{\prime \prime}-k y_{1} & =-k\left(A^{2} e^{2 \sqrt{k} x}+2 A B+B^{2} e^{-2 \sqrt{k} x}\right), \\
y_{1}(0) & =0, y_{1}(1)=0 .
\end{aligned}
$$


To solve (13), we employ the variation of parameters method (Chow 1995) which requires evaluating the following integrals

$$
u_{1}=-\int \frac{f(x) e^{-\sqrt{k} x} d x}{W}, u_{2}=\int \frac{f(x) e^{\sqrt{k} x} d x}{W},
$$

where $y_{1 h}=e^{\sqrt{k} x}$ and $y_{2 h}=e^{-\sqrt{k} x}$ are the solutions to the homogeneous differential equation

$$
y_{1 h}^{\prime \prime}-k y_{1 h}=0
$$

$W$ is the Wronskian of these two functions, given by

$$
W\left(y_{1 h}, y_{2 h}\right)=-2 \sqrt{k},
$$

and $f(x)$ is the right hand side of (13).

Substituting $f(x)$ and (16) into (14), leads to

$$
\begin{aligned}
& u_{1}=-\frac{A^{2} e^{\sqrt{k} x}}{2}+A B e^{-\sqrt{k} x}+\frac{B^{2} e^{-3 \sqrt{k} x}}{6}, \\
& u_{2}=\frac{A^{2} e^{3 \sqrt{k} x}}{6}+A B e^{\sqrt{k} x}-\frac{B^{2} e^{-\sqrt{k} x}}{2},
\end{aligned}
$$

Therefore, the solution for (13) is written, according to method of variation of parameters, as

$$
y_{1}(x)=C e^{\sqrt{k} x}+D e^{-\sqrt{k} x}-\frac{A^{2}}{3} e^{2 \sqrt{k} x}+2 A B-\frac{B^{2}}{3} e^{-2 \sqrt{k} x},
$$

applying boundary conditions $y_{1}(0)=0$ and $y_{1}(1)=0$ to (19) results

$$
\begin{aligned}
& C=\frac{A^{2}}{3}\left[\frac{e^{-\sqrt{k}}-e^{2 \sqrt{k}}}{e^{-\sqrt{k}}-e^{\sqrt{k}}}\right]-2 A B\left[\frac{e^{-\sqrt{k}}-1}{e^{-\sqrt{k}}-e^{\sqrt{k}}}\right], \\
&+ \frac{B^{2}}{3}\left[\frac{e^{-\sqrt{k}}-e^{-2 \sqrt{k}}}{e^{-\sqrt{k}}-e^{\sqrt{k}}}\right], \\
& D=\frac{A^{2}}{3}\left[\frac{e^{2 \sqrt{k}}-e^{\sqrt{k}}}{e^{-\sqrt{k}}-e^{\sqrt{k}}}\right]+2 A B\left[\frac{e^{\sqrt{k}}-1}{e^{-\sqrt{k}}-e^{\sqrt{k}}}\right] \\
&+\frac{B^{2}}{3}\left[\frac{-e^{\sqrt{k}}-e^{-2 \sqrt{k}}}{e^{-\sqrt{k}}-e^{\sqrt{k}}}\right] .
\end{aligned}
$$

By substituting (10) and (19) into (7) we obtain a first order approximation to the solution of (3), as it is shown

$$
\begin{aligned}
y(x)= & (A+\alpha C) e^{\sqrt{k} x}+(B+\alpha D) e^{-\sqrt{k} x}-\frac{A^{2} \alpha}{3} e^{2 \sqrt{k} x} \\
& +2 \alpha A B-\frac{B^{2} \alpha}{3} e^{-2 \sqrt{k} x} .
\end{aligned}
$$

We consider, as a case study, the following values for parameters: $\alpha=0.1, \alpha=1$, and $\alpha=1.5$ for $k=$ $0.1,1,5,10,20,50$, and 100 .

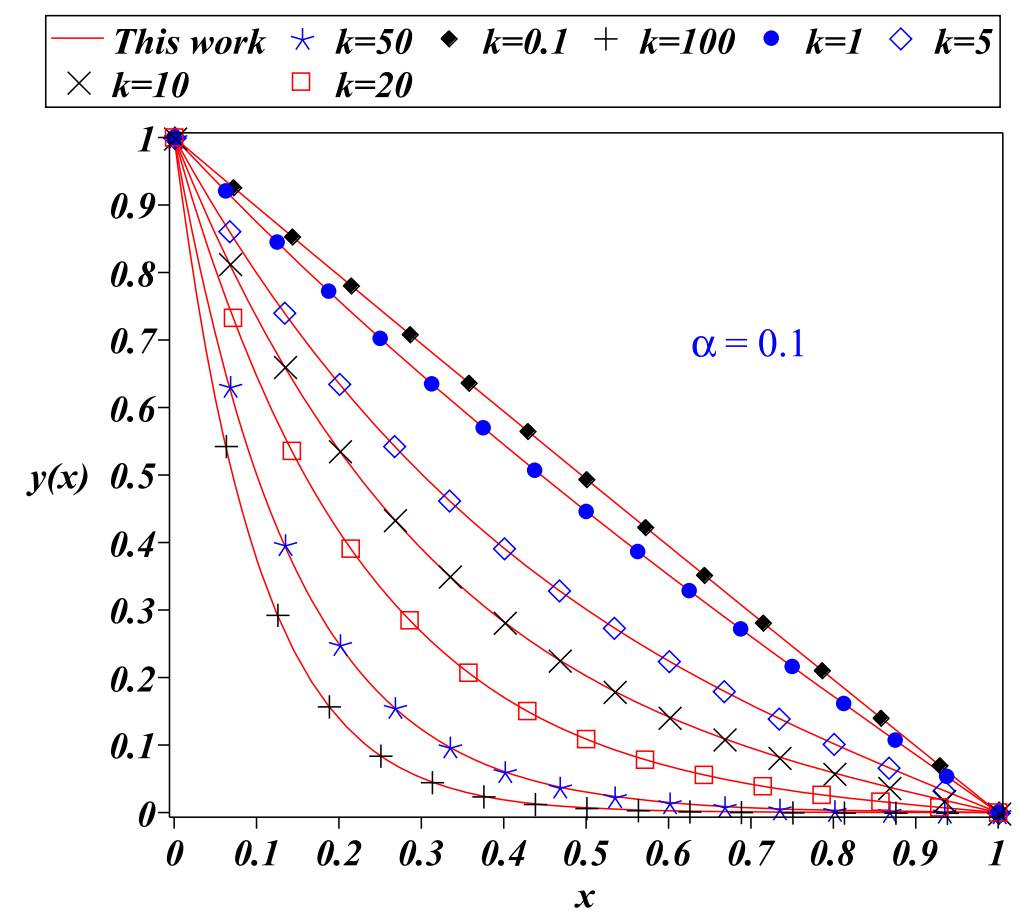

Figure 1 Fourth order Runge Kutta numerical solution for (3) (symbols) and proposed solution (20) (solid line) for $\alpha=0.1$. 


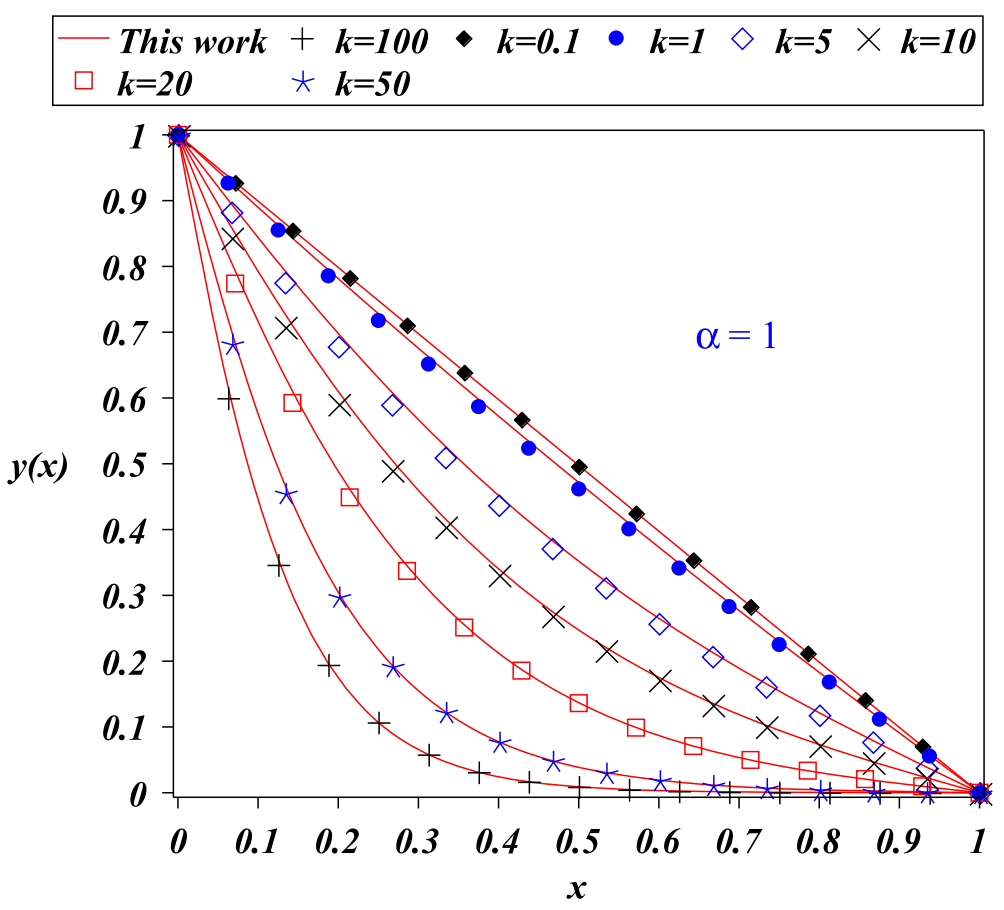

Figure 2 Fourth order Runge Kutta numerical solution for (3) (symbols) and proposed solution (20) (solid line) for $\alpha=1.0$.

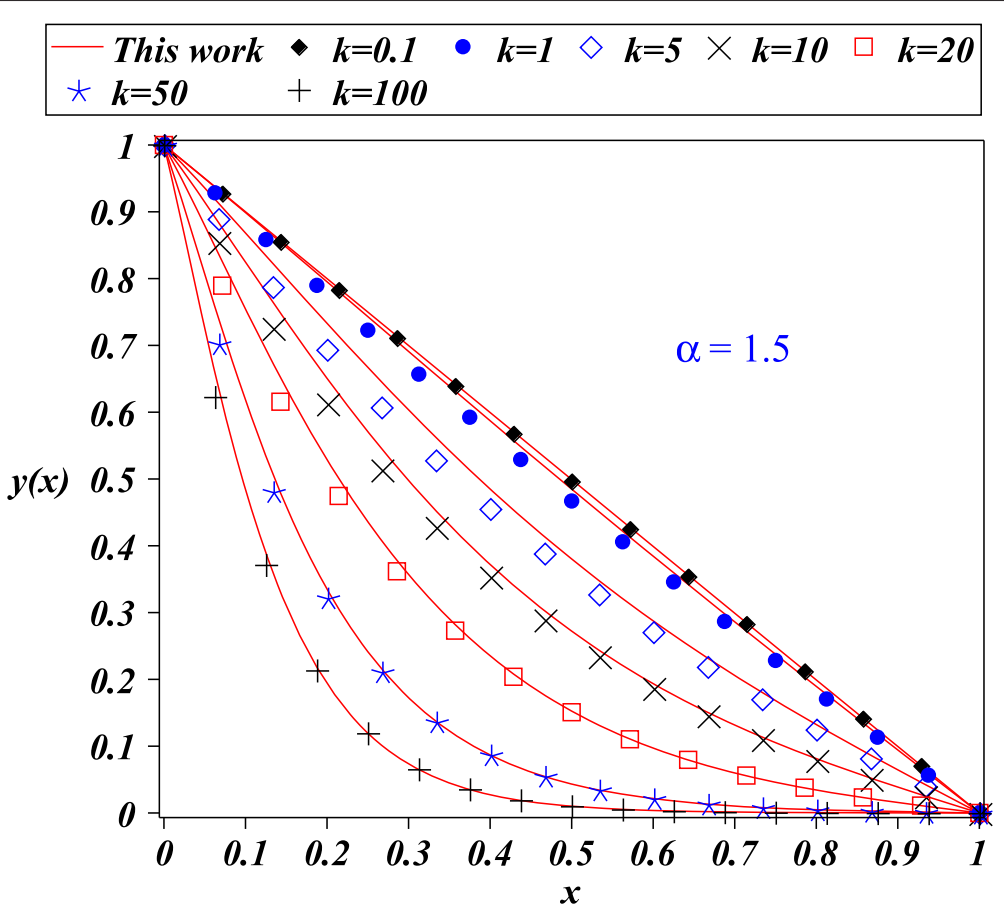

Figure 3 Fourth order Runge Kutta numerical solution for (3) (symbols) and proposed solution (20) (solid line) for $\alpha=1.5$. 


\section{Discussion}

Nonlinear phenomena appear in such broad scientific fields like applied mathematics, physics, and engineering. Scientists in those disciplines face, constantly, with the task of finding solutions for nonlinear ordinary differential equations. As a matter of fact, the possibility of finding analytical solutions for those cases is very difficult and cumbersome.

The fact that PM depends on a parameter, which is assumed to be small, suggests that the method is limited. In this work, the PM method has been applied to the problem of finding an approximate solution for the nonlinear differential equation which describes the time independent nonlinear reaction diffusion equation, corresponding to a nonlinear Michaelis-Menten kinetics scheme. This equation is relevant because its solution describes important applications such as biosensors, bioreactors, and biofuel cells, among others. Figures 1, 2, 3 show the comparison between approximation (20) for: $\alpha=0.1, \alpha=1$, and $\alpha=1.5(k=0.1,1,5,10,20,50$, and 100) to the fourth order Runge Kutta numerical solution. It can be noticed that figures are very similar for all cases, showing the accuracy of (20).

The PM method provides in general, better results for small perturbation parameters $\varepsilon<<1$ (see (1)) and when are included the most number of terms from (2). To be precise, $\varepsilon$ is a parameter of smallness; measures how much larger is the contribution of linear term $L(x)$ than $N(x)$ in (1). Although Figure 1 for $\alpha=0.1$ satisfies that condition, Figure 2 and Figure 3 show that (20) provides a good approximation as solution to (3); despite of the fact that perturbation parameters $\alpha=1$ and $\alpha=1.5$, cannot be considered small. Since that the transport and kinetics are quantified in terms of $k$ and $\alpha$, it is important that our solutions have good accuracy for a wide range of values for both parameters.

In (Thiagarajan et al. 2011), HPM method was employed to provide an approximate solution to (3). Although the solution reported has good accuracy, it is too long for practical applications. Unlike the above, (20) provides good accuracy, it is simple, and computationally more efficient.

Finally, our approximate solution (20) does not depend on any adjustment parameter, for which, it is in principle, a general expression for the exposed problem.

\section{Conclusion}

An important task is to find an analytical expression that provides a good description of the solution for the nonlinear differential equations like (3). For instance, the time independent nonlinear reaction diffusion process, corresponding to a nonlinear Michaelis-Menten kinetic scheme is adequately described by (20). This work showed that some nonlinear problems can be adequately approximated employing the PM method, even for large values of the perturbation parameter; as it was done for the problem described by (3). The success of the method for this case has to be considered as an alternative to approach other nonlinear problems; this may lead to save time and resources employed using sophisticated and difficult methods. Figures 1 thru 3 show the accuracy of the proposed solutions.

\section{Competing interests}

The authors declare that they have no competing interests.

\section{Authors' contributions}

All authors contributed extensively in the development and completion of this article. All authors read and approved the final manuscript.

\section{Acknowledgements}

We gratefully acknowledge the financial support from the National Council for Science and Technology of Mexico (CONACyT) through grant CB-2010-01 \#157024. Also, authors would like to thank Rogelio-Alejandro Callejas-Molina and Roberto Ruiz-Gomez for their contribution to this project.

\section{Author details}

${ }^{1}$ Electronic Instrumentation Faculty, Universidad Veracruzana, Cto. Gonzalo Aguirre Beltran S/N, 91000 Xalapa, Mexico. ${ }^{2}$ Electronics Department, National Institute for Astrophysics, Optics and Electronics, Luis Enrique Erro 1, 72840 Sta. Maria Tonantzintla, Mexico. ${ }^{3}$ Higher Colleges of Technology, Abu Dhabi Men's College, Abu Dhabi, United Arab Emirates. ${ }^{4}$ Department of Mathematics, Zhejiang University, 310027, Hangzhou, China. ${ }^{5}$ Micro and Nanotechnology Research Center, Universidad Veracruzana, Calzada Ruiz Cortines 455, 94292, Boca del Rio, Mexico.

Received: 19 December 2013 Accepted: 19 March 2014

Published: 27 March 2014

\section{References}

Adomain G (1988) A review of decomposition method in applied mathematics. J Math Anal Appl 135: 501-544

Assas LMB (2007) Approximate solutions for the generalized k-dv- burgers' equation by he's variational iteration method. Phys Scripta 76: 161-164

Babolian E, Biazar J (2002) On the order of convergence of adomian method. Applied Mathematics and Computation 130: 383-387

Beléndez A, Pascual C, Álvarez ML, Méndez DI, Yebra MS, Hernández A (2009) High order analytical approximate solutions to the nonlinear pendulum by he's homotopy method. Physica Scripta 79: 1-27

Biazar J, Aminikhan H (2009) Study of convergence of homotopy perturbation method for systems of partial differential equations. Comput Math Appl 58: $2221-2230$

Biazar J, Ghazvini H (2009) Convergence of the homotopy perturbation method for partial differential equations. Nonlinear Anal Real World Appl 10: $2633-2640$

Chow TL (1995) Classical Mechanics. John Wiley \& Sons Inc, New York

Chowdhury SH (2011) A comparison between the modified homotopy perturbation method and adomian decomposition method for solving nonlinear heat transfer equations. J Appl Sci 11: 1416-1420

El-Shaed M (2005) Application of he's homotopy perturbation method to volterra's integro-differential equation. Int J Nonlinear Sci Numerical Simul 6: 151-162

Evans DJ, Raslan KR (2005) The tanh function method for solving some important nonlinear partial differential equation. Int J Comput Math 82: 897-905

Fathizadeh M, Madani M, Khan Y, Faraz N, Yildirim A, Tutkun S (2011) An effective modification of the homotopy perturbation method for mhd viscous flow over a stretching sheet. J King Saud Univ Sci 25: 107-113

Faraz N, Khan Y (2011) Analytical solution of electrically conducted rotating flow of a second grade fluid over a shrinking surface. Ain Shams Eng J 2: $221-226$

Feng X, Mei L, He G (2007) An efficient algorithm for solving troesch's problem. Appl Math Comput 189: 500-507 
Fereidoon A, Rostamiyan Y, Akbarzade M, Ganji DD (2010) Application of he's homotopy perturbation method to nonlinear shock damper dynamics. Arch Appl Mech 80: 641-649

Filobello-Nino U, Vazquez-Leal H, Castaneda-Sheissa R, Yildirim A Hernandez-Martinez L, Diaz DP, Perez-Sesma A, Hoyos-Reyes C (2012a) An approximate solution of blasius equation by using hpm method. Asian J Math Stat 5: 50-59

Filobello-Nino U, Vazquez-Leal H, Pereyra-Diaz D, Perez-Sesma A, Sanchez-Orea J, Castaneda-Sheissa R, Khan Y, Yildirim A, Hernandez-Martinez L, Rabago-Bernal F (2012b) Hpm applied to solve nonlinear circuits: A study case. Appl Math Sci 6: 4331-4344

Filobello-Nino U, Vazquez-Leal H, Khan Y, Yildirim A, Jimenez-Fernandez VM, Herrera-May AL, Castaneda-Sheissa R, Cervantes-Perez J (2013) Perturbation method and laplace-padé approximation to solve nonlinear problems. Miskolc Math Notes 14: 89-101

Ganji DD, Mirgolbabaei H, Miansari M, Miansari M (2008) Application of homotopy perturbation method to solve linear and non-linear systems of ordinary differential equations and differential equation of order three. $J$ Appl Sci 8: 1256-1261

Ganji DD, Babazadeh H, Noori F, Pirouz MM, Janipour M (2009) An application of homotopy perturbation method for non linear blasius equation to boundary layer flow over a flat plate. Int J Nonlinear Sci 7: 309-404

Hassana HN, El-Tawil MA (2011) An efficient analytic approach for solving two point nonlinear boundary value problems by homotopy analysis method. Math Methods Appl Sci 34: 977-989

He JH (1999) Homotopy perturbation technique. Comput Methods Appl Mech Eng 178: 257-262

He JH (2000a) A coupling method of a homotopy technique and a perturbation technique for nonlinear problems. Int J Non-Linear Mech 35: 37-43

He JH (2006a) Homotopy perturbation method for solving boundary value problems. Physics Letters A 350: 87-88

He JH (2006b) Some asymptotic methods for strongly nonlinear equations. Int J Modern Phys B 20: 1141-1199

He JH (2007) Variational approach for nonlinear oscillators. Chaos, Solitons Fractals 34: 1430-1439

He J H (2008) Recent development of the homotopy perturbation method. Topological Methods Nonlinear Anal 31: 205-209

Holmes MH (1995) Introduction to Perturbation Methods. Springer, New York

Hossein A (2011) Analytical approximation to the solution of nonlinear blasius viscous flow equation by Itnhpm. Int Sch Res Netw ISRN Math Anal 10. DOI:10.5402/2012/957473

Jiao YC, Yamamoto Y, Dang C, Hao Y (2001) An aftertreatment technique for improving the accuracy of adomian's decomposition method. Comput Math Appl 43: 783-798

Kazemnia M, Zahedi SA, Vaezi M, Tolou N (2008) Assessment of modified variational iteration method in bvps high-order differential equations. J Appl Sci 8: 4192-4197

Khan Y, Wu Q, Faraz N, Yildirim A (2011) The effects of variable viscosity and thermal conductivity on a thin film flow over a shrinking/stretching sheet. Comput Math Appl 61: 3391-3399

Khan Y, Vazquez-Leal H, Wu Q (2013) An efficient iterated method for mathematical biology model. Neural Comput Appl 23: 677-682

Kooch A, Abadyan M (2011) Evaluating the ability of modified adomian decomposition method to simulate the instability of freestanding carbon nanotube: comparison with conventional decomposition method. J Appl Sci 11: 3421-3428

Kooch A, Abadyan M (2012) Efficiency of modified adomian decomposition for simulating the instability of nano-electromechanical switches: comparison with the conventional decomposition method. Trends Appl Sci Res 7: $57-67$

Madani M, Fathizadeh M, Khan Y, Yildirim A (2011) On the coupling of the homotopy perturbation method and laplace transformation. Math Comput Model 53: 1937-1945

Mahmoudi J, Tolou N, Khatami I, Barari A, Ganji DD (2008) Explicit solution of nonlinear zk-bbm wave equation using exp-function method. J Appl Sci 8: $358-363$

Michaelis L, Menten ML (1913) Die kinetik der invertinwirkung. Biochem. Z 49: 333-369

Mirmoradia SH, Hosseinpoura I, Ghanbarpour S, Barari A (2009) Application of an approximate analytical method to nonlinear troesch's problem. App Math Sci 3: 1579-1585
Murray JD (2002) Mathematical Biology: I. An Introduction. Springer, New York

Noor MA, Mohyud-Din ST (2009) Homotopy approach for perturbation method for solving thomas-fermi equation using pade approximants. Int Nonlinear Sci 8: 27-31

Noorzad R, Tahmasebi-Poor A, Omidvar M (2008) Variational iteration method and homotopy-perturbation method for solving burgers equation in fluid dynamics. J Appl Sci 8: 369-373

Patel T, Mehta MN, Pradhan VH (2012) The numerical solution of burger's equation arising into the irradiation of tumour tissue in biological diffusing system by homotopy analysis method. Asian J Appl Sci 5: 60-66

Sharma PR, Methi G (2011) Applications of homotopy perturbation method to partial differential equations. Asian J Math Stat 4: 140-150

Thiagarajan S, Meena A, Anitha S, Rajendran L (2011) Analytical expression of the steady-state catalytic current of mediated bioelectrocatalysis and the application of he's homotopy perturbation method. J Math Chem 49: $1727-17240$

Vanani SK, Heidari S, Avaji M (2011) A low-cost numerical algorithm for the solution of nonlinear delay boundary integral equations. J Appl Sci 11: 3504-3509

Vazquez-Leal H, Filobello-Nino U, Castaneda-Sheissa R, Hernandez-Martinez L, Sarmiento-Reyes A (2012a) Modified hpm methods inspired by homotopy continuation methods. Math Probl Eng 20. DOI: 10.1155/2012/309123

Vazquez-Leal H, Castaneda-Sheissa R, Filobello-Nino U, Sarmiento-Reyes A, Sanchez-Orea J (2012b) High accurate simple approximation of normal distribution integral. Math Probl Eng 22. DOl:10.1155/2012/124029

Xu F (2007) A generalized soliton solution of the konopelchenko-dubrovsky equation using exp-function method. Z Naturforsch 62a: 685-688

Zhang LN, Xu L (2007) Determination of the limit cycle by he's parameter expansion for oscillators in a $u^{3} / 1+u^{2}$ potential. $Z$ Naturforsch 62a: 396-398

doi:10.1186/2193-1801-3-162

Cite this article as: Filobello-Nino et al:: A handy approximation for a mediated bioelectrocatalysis process, related to Michaelis-Menten equation. SpringerPlus 2014 3:162

\section{Submit your manuscript to a SpringerOpen ${ }^{\circ}$ journal and benefit from:}

- Convenient online submission

- Rigorous peer review

- Immediate publication on acceptance

- Open access: articles freely available online

- High visibility within the field

- Retaining the copyright to your article

Submit your next manuscript at $>$ springeropen.com 